\title{
Initial letter and semantic category fluency in Alzheimer's disease, Huntington's disease, and progressive supranuclear palsy
}

\author{
Anne Rosser, John R Hodges
}

\begin{abstract}
Ten patients with dementia of Alzheimer's type, 10 patients with progressive supranuclear palsy, and 10 patients with Huntington's disease were compared on two types of verbal fluency task-namely, initial letter fluency and category (semantic) fluency. The groups were carefully matched for overall level of dementia on the dementia rating scale, and were compared with 25 age matched normal controls. The controls found letter fluency more difficult than category fluency, and this relative pattern of performance was repeated in the progressive supranuclear palsy and Huntington's disease groups, although both groups were significantly impaired on both tasks. By contrast, patients with Alzheimer's disease performed just as poorly as the progressive supranuclear palsy and Huntington's disease groups on the category tasks, but were significantly less impaired at letter fluency, performing at near normal levels on this task. From these results, it is suggested that the performances of patients with progressive supranuclear palsy and Huntington's disease relate largely to initiation and retrieval problems secondary to disruption of frontostriatal circuits, whereas in Alzheimer's disease, the poorer performance on category fluency is due principally to the breakdown of semantic knowledge, which probably reflects temporal neocortical involvement.
\end{abstract}

(F Neurol Neurosurg Psychiatry 1994;57:1389-1394)

Alzheimer's disease, which affects predominantly medial temporal and posterior cortical regions, is associated with a different pattern of cognitive impairment from dementias associated with damage to subcortical structures such as progressive supranuclear palsy, Huntington's disease, multiple sclerosis related dementia, and AIDS dementia complex. ${ }^{1-4}$ Patients with Alzheimer's disease characteristically present with deficits of memory, aphasia, or visuospatial impairment, whereas subcortical dementias produce patients who are slow, apathetic, forgetful, and have difficulty manipulating new information. ${ }^{15}$ Recent studies of patients with Alzheimer's disease using theoretically motivated neuropsychological tests have shown evidence of episodic memory impairment that is secondary to poor encoding and storage of new material, as well as an accelerated rate of forgetting. ${ }^{2}$ With disease progression there is also a breakdown of structure and organisation of semantic memory. ${ }^{6-8}$ By contrast, memory is less impaired in subcortical dementias, such as Huntington's disease, and the main difficulty seems to be the operation of effective retrieval strategies to search for information from memory stores. ${ }^{2389}$ Alzheimer's disease and subcortical types of dementia (Huntington's disease and progressive supranuclear palsy) have been shown to differ on the memory and initiation subtests of the dementia rating scale, when matched for overall level of dementia on that scale, with patients with Alzheimer's disease performing poorly on tests of memory, and patients with Huntington's disease and progressive supranuclear palsy performing poorly on tests of initiation. ${ }^{11} 12$

Disorders of language have also long been recognised as part of the symptomatology of many progressive dementias, and there are theoretical reasons to believe that different dementias may differ on tests of language production, such as letter and category fluency. Letter fluency requires subjects to generate words beginning with certain letters, whereas in category fluency tests patients are asked to generate exemplars from a given category (for example, animals, vegetables, household objects). Tests are usually conducted in the setting of a time constraint, for example, one minute per letter or category. Performance on both tasks depends upon frontostriatal circuits that control aspects of executive function (including attention, initiation, and retrieval processes) and working memory. Also, the two types of task depend on the integrity of the stores from which the examples are retrieved. In the case of initial letter fluency, the phonologically based word store is clearly critical, whereas category fluency depends on the intactness of semantic memory. ${ }^{81314} \mathrm{We}$ would argue, therefore, that frontostriatal deficits, of the type found in patients with subcortical dementias, should produce equally severe impairment on letter and category fluency tasks. By contrast, disorders that cause breakdown in the organisation of semantic memory, such as Alzheimer's disease, should result in more pronounced impairment on category fluency.

In the case of Alzheimer's disease, there has been some controversy concerning its relative impact on letter and category fluency. Whereas some workers have found disproportionate 
impairment on category fluency tests, ${ }^{3} 101315$ others have found equal impairment on both types of fluency test. ${ }^{1617}$ Recent studies that have compared groups of patients with Alzheimer's disease and Huntington's disease, matched for overall level of disease severity, have, however, lent support to the hypothesis that cortical and subcortical dementia cause differential effects on letter and category fluency. ${ }^{318}$ Before accepting the generality of this hypothesis it is important to compare patients with a wider range of diseases. Although progressive supranuclear palsy is an obvious candidate as the pathological changes are restricted, almost entirely, to subcortical structures, ${ }^{1920}$ few studies have compared patients with progressive supranuclear palsy and other diseases, and none have included both letter and category fluency tests. Milberg and Albert $^{21}$ reported a double dissociation between naming (with the Boston naming test) and letter fluency in groups of patients with Alzheimer's disease and progressive supranuclear palsy, but did not investigate category fluency in their patients. Pillon et al ${ }^{22}$ compared the performance of groups of patients with Alzheimer's disease, progressive supranuclear palsy, Huntington's disease, and Parkinson's disease on a wide ranging battery of tests including two measures of verbal fluency (animals and words beginning with $M$ ); the progressive supranuclear palsy group were more impaired than the Alzheimer's disease and Parkinson's disease groups but because the results of the two fluency tests were combined, it is not possible to comment on relative levels of performance on the two tasks across the groups.

In the study reported here, patients with Alzheimer's disease, Huntington's disease, and progressive supranuclear palsy were carefully matched for overall level of dementia on the dementia rating scale, and were also matched for age and educational level. All groups were given letter and category fluency tests and were compared with normal age matched controls. We predicted that Huntington's disease and progressive supranuclear palsy would produce equally severe impairment in letter and category fluency, and that Alzheimer's disease would produce disproportionately more impairment in category fluency.

\section{Materials and methods \\ SUBJECTS}

The study involved 55 subjects in total. Ten patients had a diagnosis of progressive supranuclear palsy according to criteria described by Lees, ${ }^{23} 10$ patients had Huntington's disease as defined by chorea, intellectual decline and a positive family history, and 10 patients had a diagnosis of probable Alzheimer's disease according to the National Institute of Neurological Disorder and Stroke and the Alzheimer Disease and Related Disease Association (NINCDSADRDA). ${ }^{24}$ The patients with Alzheimer's disease were selected from a larger cohort of about 50 patients undergoing longitudinal neuropsychological assessment to match the progressive supranuclear palsy and Huntington's disease groups in terms of age, education, and score on the Dementia Rating Scale.

Examination and laboratory tests for the differential diagnosis of dementia were carried out on all patients to exclude other causes of dementia. All patients had brain MRI or CT.

Twenty five community dwelling normal volunteers from the MRC Applied Psychology Unit's subject panel formed the normal control group. These subjects were selected to include the same age and educational range as the patient groups.

\section{NEUROPSYCHOLOGICAL TESTS}

Each subject was assessed on the Dementia Rating Scale with the standard method described by Mattis. ${ }^{25}$ Two commonly used verbal fluency tasks-letter and category fluency-were presented to all subjects in the four groups. Subjects were asked to generate as many words as possible in one minute. For letter fluency, three trials were performed with the letters F, A, and S and subjects were instructed to exclude proper nouns and the same word with different suffixes (fix, fixed, fixing etc.). For category fluency tests, subjects were given one minute for each of three living categories (animals, birds, and water creatures) and three man made categories (household objects, vehicles, and musical instruments). ${ }^{8}$

The principal score used for intergroup analysis was the total number of correct responses. For letter fluency, the summed correct responses of the three letters was used. For category fluency, the summed correct responses of the three living and three man made categories were analysed separately.

Errors were classified as perseverations (repetition of an item from earlier in the list or the same word stem with a different suffix), intrusions (the inclusion of an item from another category or, in the case of letter fluency, beginning with the wrong letter) and others (bizarre and inappropriate responses that could not be classified as one of the other error types).

\section{ANALYSES}

One way analysis of variance (ANOVA) was used to analyse differences between groups. Where main group effects were found, post hoc comparisons were made by $t$ tests with Newman-Keuls correction.

\section{DEMOGRAPHIC DATA}

Table 1 gives the demographic data. There was no significant difference between the groups in terms of age $(F(3,51)=2 \cdot 8$, $\mathrm{p}>0.05)$ or educational level $(F(3,51)=$ $0.64, p>0.05)$. The patient groups were also well matched on the Dementia Rating Scale, although as expected, the normal controls scored higher; one way ANOVA showed a significant main effect for groups $(F(3,51)=$ $24.45, p<0.0001)$ and post hoc analysis showed significant $(\mathrm{p}<0.05)$ differences 
Table 1 Age, education, and dementia rating score total scores for the controls and patients with dementia of Alzheimer's type (DAT), progressive supranuclear palsy (PSP), and Huntington's disease (HD)

\begin{tabular}{lcccc}
\hline & $\begin{array}{l}\text { Controls } \\
(n=25)\end{array}$ & $\begin{array}{l}D A T \\
(n=10)\end{array}$ & $\begin{array}{l}P S P \\
(n=10)\end{array}$ & $\begin{array}{l}H D \\
(n=10)\end{array}$ \\
\hline Age (y) (mean (SD)) & $69(8 \cdot 4)$ & $67 \cdot 2(9 \cdot 9)$ & $65 \cdot 8(8 \cdot 6)$ & $59 \cdot 9(8 \cdot 0)$ \\
Range & $53-85$ & $51-80$ & $52-82$ & $43-72$ \\
Education (y) (mean (SD)) & $10 \cdot 7(2 \cdot 3)$ & $11 \cdot 1(2 \cdot 5)$ & $10 \cdot 3(2 \cdot 6)$ & $9 \cdot 7(1 \cdot 5)$ \\
DRS (mean (SD)) & $139 \cdot 9(3 \cdot 6)$ & $121 \cdot 4(8 \cdot 3)$ & $122 \cdot 2(14 \cdot 4)$ & $121 \cdot 5(6 \cdot 2)$ \\
Range & $130-144$ & $107-132$ & $90-138$ & $112-133$ \\
\hline
\end{tabular}

Table 2 Error data for letter fluency and living category fluency tests (figures were similar for non-living categories) expressed as a percentage of total exemplars generated for the control subjects and patients with dementia of Alzheimer's type (DAT), progressive supranuclear palsy (PSP), and Huntington's disease (HD).

\begin{tabular}{|c|c|c|c|c|c|}
\hline & \multirow{2}{*}{$\begin{array}{l}\text { Letter fluency } \\
\text { perseveration } \\
(\%)\end{array}$} & \multicolumn{4}{|c|}{ Category fluency (living) } \\
\hline & & $\begin{array}{l}\text { Intrusions } \\
(\%)\end{array}$ & $\begin{array}{l}\text { Perseverations } \\
(\%)\end{array}$ & $\begin{array}{l}\text { Intrusions } \\
(\%)\end{array}$ & $\begin{array}{l}\text { Other } \\
(\%)\end{array}$ \\
\hline $\begin{array}{l}\text { Control } \\
\text { DAT } \\
\text { PSP } \\
\text { HD }\end{array}$ & $\begin{array}{l}1 \cdot 2 \\
3 \cdot 1 \\
7 \cdot 0 \\
7 \cdot 9\end{array}$ & $\begin{array}{l}0.7 \\
1.1 \\
3.2 \\
3.9\end{array}$ & $\begin{array}{l}1 \cdot 4 \\
7 \cdot 7 \\
6 \cdot 1 \\
2 \cdot 8\end{array}$ & $\begin{array}{l}0 \cdot 7 \\
2 \cdot 2 \\
2 \cdot 6 \\
1 \cdot 4\end{array}$ & $\begin{array}{l}\frac{1 \cdot 0}{0 \cdot 2} \\
2 \cdot 1\end{array}$ \\
\hline
\end{tabular}

between the patient groups and control groups, but no differences between Alzheimer's disease, Huntington's disease, and progressive supranuclear palsy groups.

\section{Results}

The figure shows results of verbal and category fluency tests in Alzheimer's disease, Huntington's disease, progressive supranuclear palsy, and normal control groups. Normal elderly subjects were able to recall a mean of 44.5 (SD 9.9) exemplars for the three letters F, A, S combined, compared with 34.8 (14.0) for Alzheimer's disease, 20.7 (12.9) for progressive supranuclear palsy, and $17 \cdot 8$ $(11 \cdot 8)$ for Huntington's disease groups. A one way ANOVA showed a highly significant

\footnotetext{
Performance of the control subjects and patients with dementia of Alzheimer's type (DAT), progressive supranuclear palsy (PSP) and Huntington's disease (HD) on letter $(F, A, S)$ and semantic category (three living and three man made categories shown separately) fluency tests showing the mean number of items correct for each group (SEM).
}

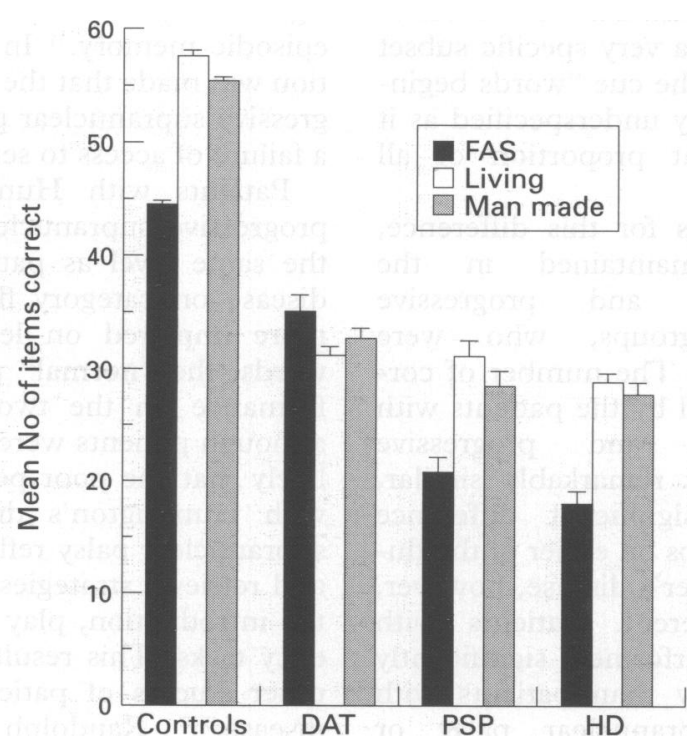

overall group effect $(F=17.55(3,51)$ p $<$ $0.0001)$. Post hoc pairwise analyses showed that the normal controls produced significantly higher scores than each of the patient groups. In addition, patients with Alzheimer's disease scored significantly higher than patients with Huntington's disease or progressive supranuclear palsy, but those with Huntington's disease or progressive supranuclear palsy were not significantly different from each other. In terms of the proportional reduction relative to the controls' mean score, the Alzheimer's disease group showed a $22 \%$ reduction whereas the progressive supranuclear palsy and Huntington's disease groups showed $54 \%$ and $60 \%$ reductions, respectively.

For category fluency, the scores for total correct living and man made items were analysed separately; however, the differences between the groups were identical for both parts of the test. As shown in previous studies, normal controls scored more highly on the category than the letter fluency tests. Again, there was a highly significant group effect for both living $(F=25.5(3,51), \mathrm{p}<0.0001)$ and man made $(F=32.71 \quad(3,51), \mathrm{p}<0.0001)$ categories. Post hoc analyses showed significant differences between normal controls (mean scores $57.4(12.7)$ and $55 \cdot 1$ (8.5) for living and man made, respectively) and all three patient groups (Alzheimer's disease $31 \cdot 1$ $(6 \cdot 1)$ and $32.5(9 \cdot 6)$ for living and man made, progressive supranuclear palsy $31 \cdot 1(14 \cdot 2)$ and $28.4(12.9)$ for living and man made, and Huntington's disease $28.8(8.5)$ and 27.7 (9.6) for living and man made). By contrast with the letter fluency tests, however, there was no significant difference between Alzheimer's disease, Huntington's disease, and progressive supranuclear palsy groups on performance in either living or man made category fluency tests. In terms of the proportional reduction, relative to the mean score of the control group, the Alzheimer's disease, progressive supranuclear palsy, and Huntington's disease groups showed reductions of $46 \%, 46 \%$, and $50 \%$ respectively for the living categories, with virtually identical figures for the man made categories.

Table 2 shows the error rates, expressed as the percentage of the number of exemplars produced for each group. For all tasks, the patient groups produced more errors than controls. On letter fluency, the rate of perseverative errors was almost twice as high for the Huntington's disease and progressive supranuclear palsy groups compared with the Alzheimer's disease group. This difference almost produced a significant group effect $(p<0.065)$. For category fluency, there was an overall group effect for perseverative errors $(F=3.88(3,51)$, p $<0.014)$ and post hoc comparisons showed that patients with Alzheimer's disease produced significantly more perseverative errors than controls or patients with Huntington's disease. There was no significant difference between patients with Huntington's disease and controls. Patients with progressive supranuclear palsy 
produced more errors than controls, but did not differ significantly from controls or patients with Alzheimer's disease. The proportions of intrusion errors were not significantly different between groups for any of the fluency tasks.

\section{Discussion}

In this study, two different types of verbal fluency task (letter and category) were given to patients with Alzheimer's disease, Huntington's disease, and progressive supranuclear palsy, all of whom had mild levels of dementia, and the scores were compared with those of normal elderly controls. Patients were well matched for overall level of dementia and other demographic details. Normal controls performed less well on letter fluency than on category fluency, a finding consistent with other studies. ${ }^{315}{ }^{18}$ It is interesting to speculate on the reasons for this difference. We assume that identical executive or supervisory processes are involved in the initiation and monitoring of both tasks, and that the difference reflects the nature of semantic, as opposed to phonological, representations, or the specificity of the retrieval cues involved in the two tasks. The representation of semantic knowledge is clearly a fundamental aspect of human cognition that has been shown to precede linguistic competence in infants. ${ }^{26}$ Furthermore, there is a considerable body of evidence that knowledge is organised by category (for example, living $v$ man made items; land animals). ${ }^{27}$ When performing any category fluency tasks it is essential to access semantic stores: activation of an initial, and usually highly prototypical exemplar (for example, cat or dog for the category "animals"), leads to automatic activation of closely related semantic neighbours. If automatic activation fails, subjects can also use more active search strategies. By contrast, letter fluency must be performed at the phonological level of word representation without reference to meaning, and the spread of activation within the phonological lexicon may proceed less rapidly than at the semantic level. In addition, the cue "animals" or "musical instruments" addresses a very specific subset of knowledge, whereas the cue "words beginning with $A$ " is relatively underspecified as it applies to a significant proportion of all known words.

Whatever the reasons for this difference, the relation was maintained in the Huntington's disease and progressive supranuclear palsy groups, who were impaired on both tasks. The number of correct exemplars produced by the patients with Huntington's disease and progressive supranuclear palsy was remarkably similar, and there was no significant difference between these two groups on either of the fluency tests. For Alzheimer's disease, however, the pattern was different. Patients with Alzheimer's disease performed significantly better on letter fluency than patients with either progressive supranuclear palsy or
Huntington's disease, although they were significantly impaired compared with controls. By contrast, patients with Alzheimer's disease were relatively more impaired on tests of category than on letter fluency $(46 \%, v 22 \%$ reduction compared with controls). This is reflected in the fact that intergroup analyses showed no difference between Alzheimer's disease, Huntington's disease, and progressive supranuclear palsy on category fluency tests. Thus the patients with Alzheimer's disease display a reversal of the normal pattern of performance. This finding is in agreement with some recent studies showing that patients with Alzheimer's disease are more impaired on category than letter fluency tests. ${ }^{3} 1314$

Our interpretation of these findings is that Alzheimer's disease causes impairment of semantic memory early in the course of the disease so that category fluency, which seems to be heavily dependent on intact semantic memory, is affected more severely than letter fluency. There is now overwhelming evidence that patients with Alzheimer's disease show impairment on a range of tests dependent on semantic memory including category fluency, picture naming, word-picture, and picturepicture matching, generation of word definitions, and identification of famous faces. ${ }^{6-8132829}$ Furthermore, recent work has established that this impairment is almost certainly due to breakdown of semantic knowledge, rather than failure to access memory stores. ${ }^{68}$ Semantic memory has not been as extensively investigated in patients with subcortical dementias but the evidence to date suggests that it does not break down to the same extent in Huntington's disease. ${ }^{3730}$ For instance, a longitudinal study by Hodges et al ${ }^{3}$ showed that semantic memory degraded more quickly in Alzheimer's disease than in Huntington's disease over the course of a year, whereas initiation and recall deteriorated more quickly in patients with Huntington's disease. A more recent comparison of patients with progressive supranuclear palsy and Alzheimer's disease failed to show differences between the two groups on tests of semantic memory, although the Alzheimer's disease group was significantly more impaired on tests of episodic memory. ${ }^{31}$ In this study the suggestion was made that the impairment in the progressive supranuclear palsy group may reflect a failure of access to semantic representations.

Patients with Huntington's disease and progressive supranuclear palsy performed to the same level as patients with Alzheimer's disease on category fluency tasks, but were more impaired on letter fluency. In other words, the "normal" pattern of relative performance on the two tasks was preserved, although patients were impaired in both. It is likely that the poor performances of patients with Huntington's disease and progressive supranuclear palsy reflects impaired initiation and retrieval strategies which, as discussed in the introduction, play a key part in both fluency tasks. This result is in accordance with other studies of patients with Huntington's disease. $^{317}$ Randolph et $a l^{32}$ investigated 
performance on a category fluency task in "uncued" and "cued" conditions in patients with Alzheimer's disease, Huntington's disease, and Parkinson's disease. They found that patients with Alzheimer's disease performed equally badly in both conditions, whereas patients with Huntington's disease or Parkinson's disease performed relatively better with cueing. On the basis of this finding, they concluded that patients with Alzheimer's disease were impaired due to degradation of semantic memory, whereas patients with Huntington's disease and Parkinson's disease fail due to impaired retrieval mechanisms rather than degradation of knowledge which explains their improved performance with cueing.

The differences in performance on letter $v$ category fluency are likely to relate to the differences in pathology in the cortical dementia of Alzheimer's disease compared with the predominantly subcortical pathology of progressive supranuclear palsy and Huntington's disease. The neural substrate of semantic memory remains unsettled but current evidence implicates the temporal neocortex as the most important region. ${ }^{33} 34$ Patients with the syndrome of semantic dementia, in which there is progressive yet selective loss of semantic memory with relative preservation of other linguistic (phonological and syntactic) and non-verbal cognitive (for example, complex perceptual and visuospatial) abilities, all have structural or functional changes in the temporal lobe(s) ${ }^{35}$; on MRI, the areas most involved seemed to be the temporal neocortex, with an emphasis on the middle and inferior temporal gyri. Similarly, patients with relatively selective loss of semantic memory after herpes simplex virus encephalitis typically show destruction of the temporal neocortex. ${ }^{36} 37$ The breakdown of the semantic memory is likely, therefore, to reflect temporal neocortical damage.

Impairment of initiation and retrieval strategies in progressive supranuclear palsy and Huntington's disease is likely to relate to damage in subcortical frontostriatal circuits. There is pathological, dynamic scanning, and neuropsychological evidence available to support this assumption. Although cortical pathology is known to occur in progressive supranuclear palsy and Huntington's disease the bulk of the neuropathological changes involve subcortical structures, at least at the early stage of the disease. ${ }^{192038}$ Positron emission tomography shows a frontal pattern of hypoperfusion in progressive supranuclear palsy, which is in keeping with the suggestion that subcortical pathology results in functional frontal deactivation. ${ }^{39} 40$ Moreover, there is now clear evidence that both progressive supranuclear palsy and Huntington's disease produce a profile of neuropsychological deficits that parallels that found in patients with frontal cortical damage. ${ }^{211122122}$ For instance, Robbins, Sahakian, and coworkers have shown that patients with progressive supranuclear palsy and Huntington's disease show pronounced impairment on their com- puterised battery of attentional and executive tasks (CANTAB), whereas patients with early Alzheimer's disease perform relatively normally on some of the same tasks from the CANTAB battery. ${ }^{41-43}$ Other frontal lobe syndromes have been shown to impair verbal fluency in a similar way: a non-aphasic patient with trauma induced bilateral frontal lobe lesions, described by Randolph et $a l,{ }^{29}$ was impaired on verbal fluency tests but improved to normal after cueing, suggesting that the prefrontal cortex may be critical in maximising the search strategies. Similar results were obtained in a detailed single case study of a patient with frontal Pick's disease who exhibited a severe reduction in letter and category fluency in the absence of any evidence of semantic memory impairment on other measures. ${ }^{44}$ As Pick's disease is a cortical disorder, but one which produces a "subcortical" profile of behavioural and neuropsychological deficits, it would perhaps be better to adopt the term "frontostriatal dementias" to encompass patients with both subcortical and progressive frontal neurodegenerative diseases.

In summary, we suggest that the differential performance of patients with Alzheimer's disease compared with those with Huntington's disease and progressive supranuclear palsy, on tests of verbal fluency is due to breakdown of semantic knowledge structure in Alzheimer's disease and to the disruption of subcortical-frontal circuits in progressive supranuclear palsy and Huntington's disease, and that these differences relate to the differences in underlying pathology.

This research is supported in part by an MRC project grant to JRH. We thank Naida Graham for her help with the statistical analysis.

1 Cummings JL. Subcortical dementia. Oxford: Oxford University Press, 1990.

2 Heindel WC, Salmon DP, Butters N. Cognitive approaches to the memory disorders of demented patients. In: Sutker PB, Adams HE, eds. Comprehensive textbook of psychopathology. 2nd ed. New York: Plenum Press, 1993.

3 Hodges JR, Salmon DP, Butters N. Differential impairment of semantic and episodic memory in Alzheimer's and Huntington's disease: a controlled prospective study. F Neurol Neurosurg Psychiatry 1990;53:1089-95.

4 Moss MB, Albert MS, Butters N, Payne M. Differential patterns of memory loss among patients with patterns of memory loss among patients with Korsakoff's syndrome. Arch Neurol 1986;43:239-46.

5 Albert ML, Feldman RG, Willis AL. The "subcortical dementia" of progressive supranuclear palsy. $₹$ Neurol Neurosurg Psychiatry 1974;37:121-30.

6 Chertkow H, Bub D. Semantic memory loss in dementia of Alzheimer type. What do various measures measure? Brain 1990;113:397-417.

7 Hodges JR, Salmon DP, Butters N. The nature of the naming deficit in Alzheimer's and Huntington's Disease. Brain 1991;114:1547-58.

8 Hodges JR, Salmon DP, Butters N. Semantic memory impairment in Alzheimer's disease: failure of access or degraded knowledge? Neuropsychologia 1992;30:301-14.

9 Butters N, Wolfe J, Martone M, Granholm E, Cermak LS. Memory disorders associated with Huntington's disease: verbal recall, verbal recognition and procedural memory. Neuropsychologia 1985;23:729-43.

10 Butters N, Wolfe J, Granholm E, Cermak LS. An assessment of verbal recall, recognition and fluency abilities in patients with Huntington's disease. Cortex 1986;22: $11-32$.

11 Rosser AE, Hodges JR. The dementia rating scale in Alzheimer's disease, Huntington's disease and progressive supranuclear palsy. $\mathcal{F}$ Neurol 1994 (in press).

12 Salmon DP, Kwo-on-Yuen PF, Heindel WC, Butters N, Thal LJ. Differentiation of Alzheimer's disease and Huntington's disease with the Dementia Rating Scale. Arch Neurol 1989;46:1204-6.

13 Martin A, Fedio P. Word production and comprehension in Alzheimer's disease: the breakdown of semantic knowledge. Brain Lang 1983;19:124-41. 
14 Butters N, Granholm E, Salmon DP, Grant I, Wolfe J. Episodic and semantic memory: a comparison of amnesic and demented patients. $\mathcal{f}$ Clin Exp Neuropsychol 1987;9:479-97.

15 Monsch AU, Bondi MW, Butters N, Salmon DP, Katzman R, Thal LJ. Comparisons of verbal fluency
tasks in the detection of dementia of the Alzheimer type. tasks in the detection of dement

16 Rosen W. Verbal fluency in aging and dementia. $f$ Clin Neuropsychol 1980;2:135-46.

17 Ober BA, Koss E, Friedland RP, Delis DC. Processes of verbal memory failure in Alzheimer'-type dementia. Brain Cogn 1985;4:90-103.

18 Monsch AU, Bondi MW, Butters N, Paulsen JS, Salmon DP, Brugger P, Swenson MR. A comparison of category and letter fluency in Alzheimer's disease

19 Jellinger KA, Bancher C. Neuropathology. In: Litvan I, Agid Y, eds. Progressive supranuclear palsy: clinical and research approaches. New York: Oxford University Press, research approact

20 Steele JC, Richardson JC, Olszewski J. Progressive Supranuclear Palsy: A heterogeneous degeneration involving the brain stem, basal ganglia, and cerebellum, involving the brain stem, basal ganglia, and cerebellum,
with vertical gaze and pseudobulbar palsy, nuclear dyswith vertical gaze and pseudobulbar palsy, nuclear
tonia, and dementia. Arch Neurol 1964;10:333-59.

21 Milberg W, Albert $M$. Cognitive differences between patients with progressive supranuclear palsy and Alzheimer's disease. $\mathcal{F}$ Clin Exp Neuropsychol 1989;11: 605-14.

22 Pillon B, Dubois B, Ploska A, Agid Y. Severity and specificity of cognitive impairment in Alzheimer's, Huntington's, and Parkinson's diseases, and Progressive Supranuclear Palsy. Neurology 1991;41:634-43.

23 Lees AJ. The Steele-Richardson-Olszewski syndrome. In Marsden CD, Fahn S, eds. Movement disorders 2. London: Butterworths, 1987:272-87.

24 McKhann G, Drachman D, Folstein M, et al. Clinical diagnosis of Alzheimer's disease: report of the NINCDS-ADRDA Work Group under the auspices of Department of Health and Human Services Task Force on Alzheimers Disease. Neurology 1984;34:939-44.

25 Mattis S. Dementia rating scale. Windsor: NFER-Nelson, 1992.

26 Mar 'ler JM, Bauer PJ, McDonough J. Separating the sheep from the goats: Differentiating global categories. Cogn Psychol 1991;23:263-98.

27 Shalice T. From neuropsychology to mental structure. Cambridge: Cambridge University Press, 1988.

28 Bayles KA, Tomoeda CK. Confrontational naming impairment in dementia. Brain Lang 1983;19:98-114.

29 Hodges JR, Patterson KP. Is semantic memory consistently impaired early in the course of Alzheimer's disease?: neuroanatomical and diagnostic implications. Brain 1994 (in press).
30 Smith S, Butters N, White R, Lyon L, Granholm E. Priming semantic relations in patients with Huntington's disease. Brain Lang 1988;33:27-40.

31 van der Hurk $P$, Hodges JR. Episodic and semantic memory in Alzheimer's disease and progressive supranuclear palsy: a comparative study. F Clin Exp Neuropsychol 1994 (in press).

32 Randolph C, Braun AR, Goldberg TE, Chase TN Semantic fluency in Alzheimer's, Parkinson's, and Huntington's disease: Dissociation of storage and retrieval failures. Neuropsychology 1993;7:82-88.

33 Damasio AR, Damasio H, Tranel D, Brandt JP. Neural regionalization of knowledge access: preliminary evidence. Symposia on quantitative biology. Cold Spring Habour Laboratory Press 1990;55:1039-47.

34 Patterson KP, Hodges JR. Disorders of semantic memory. In: Baddeley A, Wilson B, Watts F, eds. Handbook of memory disorders. Chichester: Wiley 1994 (in press).

35 Hodges JR, Patterson K, Oxbury S, Funnell E. Semantic dementia: Progressive fluent aphasia with temporal lobe atrophy. Brain 1992;115:1783-806.

36 Pietrini V, Nertempi P, Vaglia A, Revello MG, Pinna V, Ferro-Milone F. Recovery from herpes simplex encephalitis: selective impairment of specific semantic categories with neuroradiological correlation. $f$ Neurol Neurosurg Psychiatry 1988;51:1284-93.

37 Warrington EK, Shallice T. Category specific semantic impairments. Brain 1984;107:829-54.

38 Roos RAC. Neuropathology of Huntington's disease. In Vinken GW, Bruyn GW, Klawans HL, eds. Handbook of clinical neurology. Vol 49. Amsterdam: Elsevier, 1986.

39 Parks RW, Haxby JV, Grady CL. Positron emission tomography in Alzheimer's disease. In: Parks RW, Zec RF, Wilson RS, eds. Neuropsychology of Alzheimer's disease and other dementias. New York. Oxford University Press, 1993:459-88.

40 Goffinet AM, De Volder AG, Gillian C, et al. Positron emission tomography demonstrates frontal like hypometabolism in progressive supranuclear palsy. Ann Neurol tabolism in progres

41 Sahakian BJ, Downes J, Eagger S, et al. Sparing of attentional relative to mnemonic function in a subgroup of patients with dementia of the Alzheimer's type. Neuropsychologia 1990;28:1197-213.

42 Sahakian BJ, Owen AM. Computerized assessment in neuropsychiatry using CANTAB. $\mathcal{F} R$ Soc Med 1992;85 399-402.

43 Robbins TW, James M, Owen AM, et al. Cognitive deficits in progressive supranuclear palsy, Parkinson's disease and multiple system atrophy in tests sensitive to fronta lobe dysfunction. F Neurol Neurosurg Psychiatry 1994:57: 79-88.

44 Hodges JR, Gurd J. Remote memory and lexical retrieval in a case of frontal Pick's disease. Arch Neurol 1994;51: 821-7. 